\title{
Blood rheology in marine mammals
}

\author{
Michael A. Castellini ${ }^{1 *}$, Oguz Baskurt ${ }^{2}$, Judith M. Castellini ${ }^{1}$ and Herbert J. Meiselman ${ }^{3}$ \\ 1 Institute of Marine Science, University of Alaska Fairbanks, Fairbanks, AK, USA \\ 2 Department of Physiology, School of Medicine, Koc University, Istanbul, Turkey \\ ${ }^{3}$ Department of Physiology and Biophysics, Keck School of Medicine, University of Southern California, Los Angeles, CA, USA
}

Edited by:

Warren Burggren, University of North

Texas, USA

Reviewed by:

Warren Burggren, University of North

Texas, USA

Eugene Williams, Salisbury State

University, USA

${ }^{*}$ Correspondence:

Michael A. Castellini, Institute of

Marine Science, University of Alaska

Fairbanks, Fairbanks, AK 99775, USA.

e-mail:mikec@ims.uaf.edu
The field of blood oxygen transport and delivery to tissues has been studied by comparative physiologists for many decades. Within this general area, the particular differences in oxygen delivery between marine and terrestrial mammals has focused mainly on oxygen supply differences and delivery to the tissues under low blood flow diving conditions. Yet, the study of the inherent flow properties of the blood itself (hemorheology) is rarely discussed when addressing diving. However, hemorheology is important to the study of marine mammals because of the critical nature of the oxygen stores that are carried in the blood during diving periods. This review focuses on the essential elements of hemorheology, how they are defined and on fundamental rheological applications to marine mammals. While the comparative rationale used throughout the review is much broader than the particular problems associated with diving, the basic concepts focus on how changes in the flow properties of whole blood would be critical to oxygen delivery during diving. This review introduces the reader to most of the major rheological concepts that are relevant to the unique and unusual aspects of the diving physiology of marine mammals.

Keywords: hemorheology, diving, red blood cells, viscosity

\section{INTRODUCTION}

During diving, blood flow in marine mammals is highly regulated and, depending on the nature of the dive, some organs may receive extremely low blood flow for long periods. Some central tissues remain perfused even during long dives, while others can be held essentially ischemic. The regulation and control of the peripheral and central circulations during diving has been studied extensively for over 60 years and while the particulars of those control mechanisms are not discussed here, the net results are relevant to this review: Marine mammals demonstrate a suite of circulatory adjustments for the regulation of blood oxygen supply during dives. Some of these are dynamic (e.g., bradycardia, vasoconstriction, hematocrit (Hct) regulation) and others are systemic (e.g., elevated myoglobin concentrations, blood volume to body mass ratios, hemoglobin and red blood cell (RBC) concentrations). Depending on the intensity and duration of the dive response, the associated bradycardia and circulatory re-routing are adjusted with the possibility for extremely low blood flow rates in some organs (Scholander, 1940; Zapol et al., 1979; Butler, 1982; Elsner and Gooden, 1983; Kooyman, 1989). Further, in species that demonstrate splenic sequestration of RBC, Hct can increase from as low as 35\% during breathing (eupneic) periods up to $75 \%$ during diving and breath-holding (apnea) (Castellini et al., 1986, 1988; Qvist et al., 1986). Therefore, blood flow rates, $\mathrm{RBC}$ aggregation, and blood viscosity become critical parameters during diving periods.

The central questions addressed in this review are whether there are intrinsic characteristics of marine mammal blood that are adapted to maintaining flow during diving periods of variable $\mathrm{Hct}$ and if there are blood adaptations to periods of low blood flow (stasis). For example, are there rheological properties of marine mammal blood that offset the impacts of elevated Hct and low flow rates?

\section{WHAT IS "BLOOD RHEOLOGY"?}

In its broadest sense, blood rheology, usually termed hemorheology, is the study of how blood deforms and flows in both in vitro testing devices and through the circulatory system; it deals with flow characteristics of blood as a suspension of particles, with RBC-RBC interactions and with interactions of RBC with other cells and with blood vessels. The essential concepts include the viscosity of blood as a function of the number, shape, size and mechanical characteristics of $\mathrm{RBC}$, the effects of shear rate(proportional to the rate of blood flow in vessels), interactions between blood cells (RBC aggregation), the viscous properties of plasma and the geometry and properties of blood vessels. Some of these parameters can be directly measured in vitro, including plasma and blood viscosity at various Hct levels, shear rates, and temperatures (Baskurt et al., 2007). These in vitro measurements are most applicable to studies on marine mammals because they are readily adapted to blood samples collected from different species under a variety of field or laboratory conditions. For example, the impact of temperature on the viscosity of Arctic bowhead whale blood demonstrated that at the low temperatures most likely seen in the fluke, whale blood was less viscous than human blood would be under the same cool conditions (Elsner et al., 2004).

Conversely, in vivo studies that examine blood while interacting with blood vessels in a living animal are not as easily adapted to diving mammal investigations because of the complex surgical methods and specialized equipment required. Consequently, we know of no studies that have examined in vivo hemorheological properties in a marine mammal, diving or resting. While we will discuss some of these in vivo issues, our primary focus in this review will be on how comparative physiologists can utilize collected blood samples to gain insights into the interesting adaptations that characterize the circulatory system of diving mammals. 
Despite the abovementioned current absence of in vivo blood flow observations, it is our hope that this review will provide insight into why hemorheology has an important role in diving physiology. Its goal is to present to the reader some of the fundamental aspects of blood flow behavior, introduce essential rheological concepts, and describe how these can be applied to diving mammals.

In this review, we utilize the extensive and well-studied field of human hemorheology for comparative data with marine mammals. For the reader interested in even broader comparative rheology information, there are there are many excellent reviews that cover the details of the development and molecular biology of hemorheology, the effects of disease, and comparative hemorheological data (Chien et al., 1971; Chien, 1975; Hawkey, 1975; SowemimoCoker et al., 1989; Gascoyne and Hawkey, 1992; Johnn et al., 1992; Meiselman, 1993; Stoltz et al., 1999; Windberger et al., 2003; Baskurt et al., 2007; Windberger and Baskurt, 2007).

\section{BACKGROUND RHEOLOGICAL CONSIDERATIONS}

We discuss here a suite of factors that should theoretically impact the rheological properties of marine mammal blood, including: shear rate and the tendency for RBC aggregation; shape/deformability of RBC; Hct variability; and the cellular rheological behavior of RBC.

Two of the fundamental concepts in rheology require some preliminary discussion: Newtonian fluids and shear rate. Newtonian fluids are often described as "simple" fluids (e.g., water, salt solutions). For these fluids, viscosity changes are driven only by temperature alterations: viscosity decreases with increasing temperature but is not affected by flow conditions. In contrast, non-Newtonian fluids (e.g., whole blood) are complex fluids where the inherent properties of the fluid alter the viscosity depending not only on temperature but also on shear rate. Shear itself can best be described as visualizing the stream lines or layers that form as a fluid is flowing through a tube. Since the velocity of flow is zero at the wall and increases toward the center, layers move at different speeds. The difference of speed between adjacent streamlines or layers divided by the distance separating these layers defines the rate at which the layers are being "sheared" against one another and hence shear rate. The units of shear rate are unusual (i.e., inverse time, $\mathrm{s}^{-1}$ ) and arise due to a velocity term such as $\mathrm{cm} / \mathrm{sec}$ being divided by a distance such as $\mathrm{cm}$ leaving time in the denominator. Note that for a given size tube, shear rate increases with volumetric flow rate.

\section{Shear rate effects}

To illustrate how flow impacts viscosity, Figure 1 presents typical viscosity-shear rate results for three human $\mathrm{RBC}$ suspensions, all at a normal Hct of 40\%: (1) RBC in plasma; (2) RBC in isotonic buffer; (3) RBC made extremely rigid by fixation in glutaraldehyde and suspended in isotonic saline buffer. Dealing first with the RBC-plasma suspension, the feature most notable is the strong curvilinear effect of shear rate on viscosity with an 11-fold decrease in blood viscosity as shear rate increases over the range shown. Hence unlike simple fluids, blood behaves as a non-Newtonian fluid whose viscosity decreases with increasing rates of shear. At sufficiently high shear rates (i.e., $\sim 1000 \mathrm{~s}^{-1}$ ) blood viscosity becomes essentially independent of shear rate and thus exhibits Newtonian flow behavior; the process is reversible such that viscosity will again

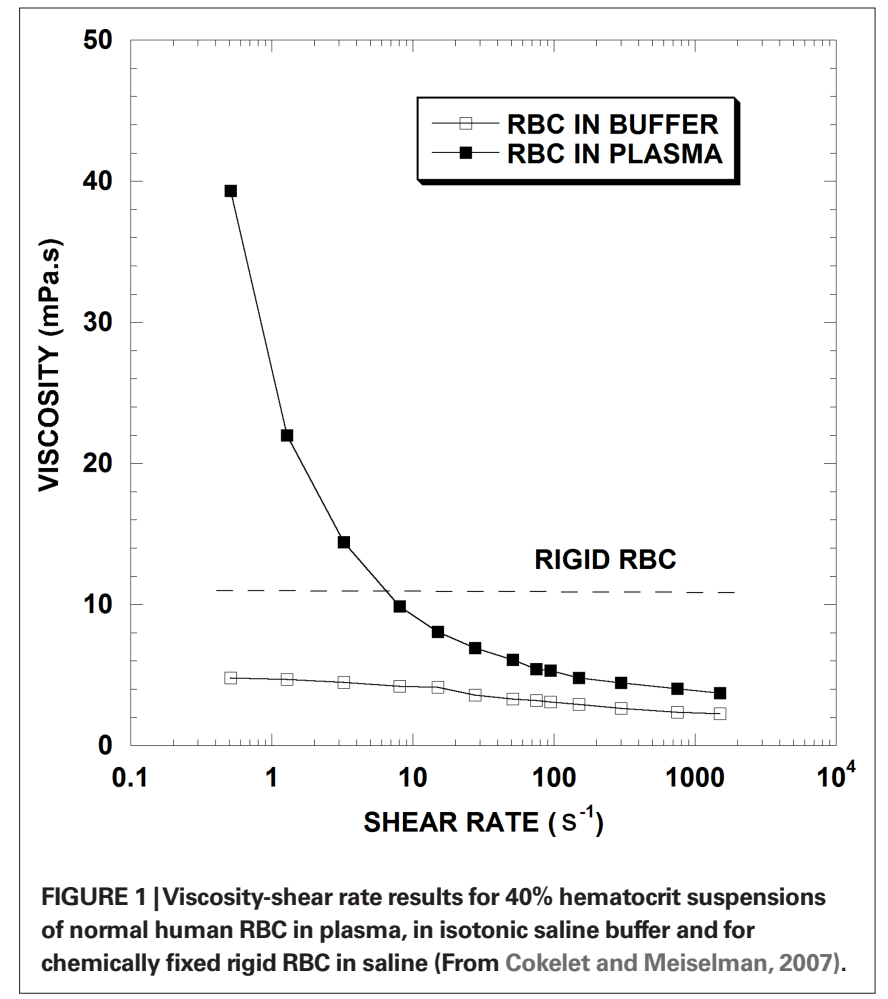

increase as shear rate is decreased. The exact shear rate necessary for Newtonian flow is Hct dependent, with higher shear needed at higher Hcts (Cokelet and Meiselman, 2007).

In contrast, the RBC-saline suspension is much less shear dependent, with less than a 2-fold decrease over the range of shear rate, and Newtonian flow appearing well prior to $1,000 \mathrm{~s}^{-1}$. Since normal RBC in plasma undergo reversible aggregation (termed "rouleaux formation") while those in saline buffer do not, RBC aggregation is considered the primary determinant of elevated blood viscosity at low shear rates. With increasing shear forces, the RBC aggregates in the plasma suspension become progressively dispersed and the viscosities of the buffer and plasma suspensions approach each other; RBC aggregation is thus the reason for blood's marked non-Newtonian behavior. Note that since plasma has a higher viscosity than buffer, the two curves in Figure 1 will not coincide unless the viscosities of plasma and buffer are equalized (Chien, 1975; Stoltz et al., 1999; Baskurt et al., 2004; Cokelet and Meiselman, 2007).

\section{Hematocrit effects}

Not surprisingly, the fraction of particles in a suspension affects its rheological behavior and in blood, this fraction is represented primarily by the Hct. Unless aggregated, platelets are too few in number and volume to affect viscosity, and white cells only influence viscosity when their volume fraction is abnormally elevated. Figure 2 presents blood viscosity data in human blood for Hcts of $20,30,40$ and $50 \%$ over a 200 -fold range of shear rate (i.e., $\left.0.1-20 \mathrm{~s}^{-1}\right)$. This figure demonstrates several principles that are relevant to this discussion: First, increases in Hct increase viscosity at all flow rates. Second, the relationship between Hct and viscosity is not linear: at the lowest shear rate shown, a Hct change from 20 to 


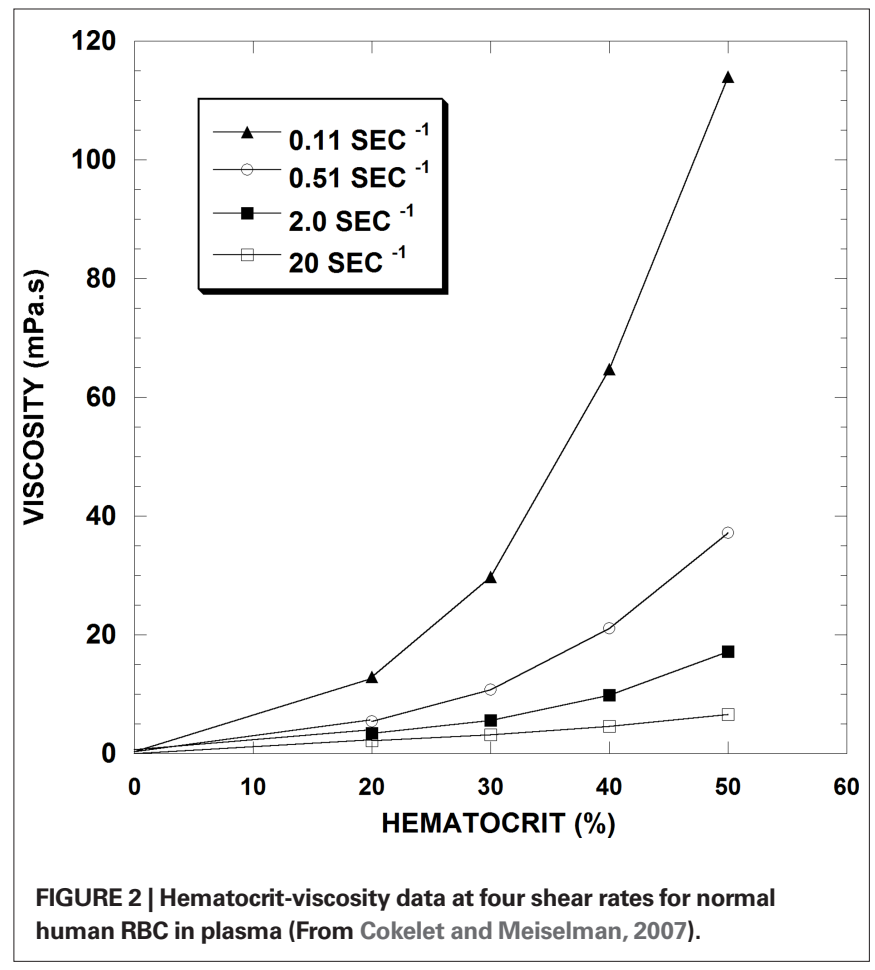

$50 \%$ increases blood viscosity by about 9-fold whereas at the highest shear rate, the same Hct change only causes a 3-fold increase. Finally, the viscosity response to shear rate is not linear, that is, the blood does not behave as a Newtonian fluid at the tested flow rates: at $20 \%$ Hct there is a 6 -fold decrease in viscosity over $0.1-20 \mathrm{~s}^{-1}$ whereas at $50 \% \mathrm{Hct}$, the decrease is about 20 -fold.

\section{RBC deformability}

Next, we consider the impacts of cell rigidity on viscosity as shown in Figure 1. Unlike normal cells, rigid RBC are unable to deform in response to shear forces and have a higher viscosity compared to normal cells at shear rates above about $10 \mathrm{~s}^{-1}$ that is best ascribed to the decrease in RBC deformability. There is general agreement that the ability of the RBC to deform is influenced by the surface area to volume ratio, cell shape, membrane mechanical properties and their internal viscosity (Chien, 1987; Baskurt and Meiselman, 2003).

\section{RBC shape and geometry}

Mammalian erythrocytes are enucleated cells and, in general, have a biconcave, discoid shape, yet RBC size and volume vary widely (Gascoyne and Hawkey, 1992). There does not always seem to be an association between the size of the animals and its RBC volume. For example, RBC mean cell volume (MCV) of mammals ranges from about $20 \mathrm{fL}$ in goat, 42 for horses, 80-95 for humans, 155 for Weddell seals, 180 in elephant seals and bowhead whales, 138 in elephants and $200 \mathrm{fL}$ in echidna (Wickham et al., 1989, 1990; Castellini et al., 2006; Baskurt et al., 2010). Table 1 presents data for further comparative MCV values in marine mammals. Interestingly, despite this wide variation in erythrocyte sizes among mammals, Hct at rest is relatively constant, with a nominal range of about

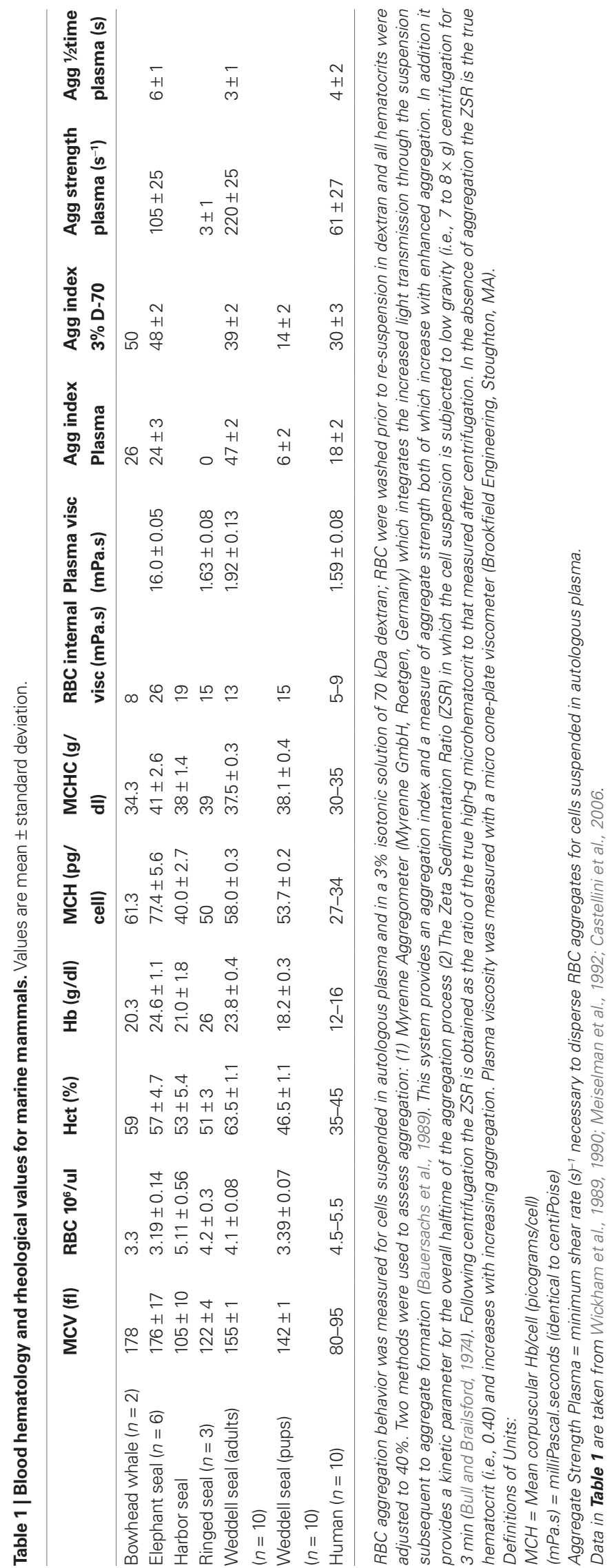


$33-45 \%$. Therefore, the number of erythrocytes per unit volume of blood also varies widely to maintain Hct values within a narrow range under resting conditions (Wickham et al., 1989; Gascoyne and Hawkey, 1992; Meiselman et al., 1992; Castellini et al., 2006).

RBC geometric characteristics such as shape and membrane surface area to cell volume ratio ( $\mathrm{S} / \mathrm{V}$ ratio) can also affect the flow behavior of blood. Shear forces can induce reversible changes of RBC shape at constant cell volume (e.g., bending, folding, irregular deformation). However, the ability of the cell to deform depends critically on its S/V ratio: a sphere has the minimum surface area for the contained volume, and thus to deform a sphere it is necessary to enlarge its area. The RBC membrane is extremely resistant to increases of area and undergoes rupture and hemolysis if enlarged more than $2-3 \%$.

\section{RBC membrane composition}

To investigate factors involved in RBC deformability, membrane components have been manipulated in various terrestrial mammal species. Modifications of RBC membrane lipid content and proteins have been associated with RBC shape changes (Wang, 1994; Wong, 1999). Membrane lipids differ among some species (Oulevey et al., 1977; Engen and Clark, 1990), but the role of dietary fatty acid intake on RBC membrane lipid content in this context is not conclusive. In guinea pigs, RBC deformability remains unchanged in spite of a $50 \%$ decrease of membrane cholesterol after statin treatment (Uyuklu et al., 2007). Such studies indicate that differences in membrane lipid structures do not satisfactorily explain species specific differences in RBC deformability.

\section{RBC cytoplasmic viscosity}

Another important parameter affecting blood rheology is RBC cytoplasmic viscosity and its effect on the rheologic behavior of red cells. Erythrocyte cytoplasmic viscosity is related to mean cellular hemoglobin concentration (MCHC), and increases faster than exponentially as MCHC rises. The increase of viscosity with hemoglobin concentration is particularly abrupt at high MCHC levels: an increase from the human norm of 32 to $41 \mathrm{~g} / \mathrm{dl}$ as seen in some seals results in a 4 -fold increase of cytoplasmic viscosity (Meiselman et al., 1992). Further, there is some evidence that elevated hemoglobin levels result in hemoglobin-cytoskeletal interactions leading to increased rigidity of the RBC membrane (Nash and Meiselman, 1983). Table 1 contains data for marine mammal MCHC and RBC (internal) cytoplasmic viscosity.

\section{RBC aggregation effects}

Marked differences of RBC aggregation and of "RBC aggregability" can greatly affect the flow behavior of blood, especially at lower shear or flow rates. Note that the two terms are not interchangeable: (1) aggregation refers to the measured extent, strength or rate of aggregation for cells in any medium including plasma; (2) the term "aggregability" has been coined to describe RBC aggregation tendency for cells that have been separated from autologous plasma, washed, and then re-suspended in a pro-aggregating, well- defined protein or polymer solution. RBC aggregability is thus an intrinsic cellular property and hence enhanced aggregation in such a solution would indicate greater RBC aggregability. This cellular property can be affected by cell deformability or morphology and is usually governed by the physicochemical characteristics of the plasma membrane carbohydrate structure (glycocalyx). An example of differing aggregability is the greater tendency for aggregation of older, more-dense cells versus younger, less-dense human RBC (Neu and Meiselman, 2007).

As mentioned above, RBC aggregation impacts hemorheological behavior because as the RBC form into multi-cell groups ("rouleaux") they change the flow characteristics of whole blood. Both aggregation and aggregability depend on cell deformability since aggregate formation requires parallel membrane surfaces between cells. We have recently reviewed some of the background and current research directions in RBC aggregation studies and noted a wide range of $\mathrm{RBC}$ aggregation properties across terrestrial mammals (Baskurt and Meiselman, 2007, 2010; Meiselman et al., 2007). Reasons for this diverse aggregation behavior include variations in RBC deformability and membrane surface properties (Eylar et al., 1962; Meiselman, 1993; Rampling et al., 2004) as well as quantitative and qualitative differences in plasma protein concentrations (Andrews et al., 1991; Kraft and Dürr, 1997). Table 1 contains data for marine mammal aggregation values.

\section{Summary}

To summarize these baseline hemorheological principals, whole blood usually behaves as a non-Newtonian fluid, with both viscosity and the degree of Newtonian behavior a function of Hct (Figure 2). This behavior is generally ascribed to two processes: (1) RBC aggregates form at stasis or low shear rates; (2) RBC aggregates are dispersed by increasing fluid shear forces and RBC undergo deformation and alignment with flow at medium to high shear rates. Thus, as shown in Figure 1, when studied at constant Hct, increases of viscosity at low shear usually implies enhanced RBC aggregation while increases at high shear are ascribed to decreased RBC deformability.

\section{MARINE VERSUS TERRESTRIAL MAMMALS}

We know that diving and breath-holding can induce large changes in Hct in marine mammals and that blood flow is variable and can be static in some organ beds. Marine mammal blood cells can also contain significantly larger amounts of hemoglobin than terrestrial mammals (Kooyman, 1989; Elsner and Meiselman, 1995; Castellini et al., 2006) and must endure rapid and massive changes in hydrostatic pressure during deep diving. This section focuses on some of these hemorheological challenges and possible adaptations in marine versus terrestrial mammals. While no in vivo studies focusing on rheological changes during diving have been conducted, there have been several in vitro examinations of marine mammal RBC.

\section{BALANCING OXYGEN SUPPLIES, HEMATOCRIT AND BLOOD VISCOSITY}

The first comparative values on marine mammal hemorheology were published by Guard and Murrish (1975). They reported that at $50 \%$ Hct, blood from Weddell, leopard and crab eater seals exhibited viscosities greater than human blood at high shear rates. It was not until over 10 years later that further rheological studies on marine mammal blood were conducted. One of the first of these was by Hedrick et al. (1986) who measured several fundamental rheological characteristics in northern elephant seal blood and, 
several years later, conducted another survey of seven marine mammal species (Hedrick and Duffield, 1991). They concluded that across species, viscosity increased exponentially with Hct, but could not find any adaptive changes to reduce whole blood viscosity. They suggested therefore that optimum Hct levels would be at about $50 \%$ in order to balance oxygen delivery with the costs of increased blood viscosity.

Wickham et al. $(1989,1990)$ also conducted experiments on harbor, ringed and elephant seal blood. They confirmed that viscosity and Hct were related, but showed that marine mammal blood was up to $28 \%$ less viscous than pig blood when measured at the same Hct and shear rates of 2-10 s $\mathrm{s}^{-1}$. Further, they demonstrated that RBC aggregation, erythrocyte sedimentation rates and plasma fibrinogen levels were lower than humans for these several seal species. On the basis of their work, it appeared that there might be a pattern such that seal blood was less viscous than human blood and that this would be a clear advantage during periods of low blood flow while diving. During this same general period however, the first evidence of variable $\mathrm{Hct}$ and blood temperature during diving was published (Kooyman et al., 1980; Qvist et al., 1986), thus adding the increased complexity that these parameters, both of which impact blood viscosity, were not constant during breath-hold periods.

Several years later, Meiselman et al. (1992) and Elsner and Meiselman (1995) sought to extend this reduced blood viscosity theory to Weddell seals, a species known for long duration diving. Surprisingly, they found that when tested at $40 \% \mathrm{Hct}$, Weddell seal blood was more viscous than ringed seal, elephant seal and human blood at all shear rates. By contrast, ringed seal blood exhibited extremely low viscosity and aggregation indices. At about the same time, it was postulated that Weddell seals sequestered RBC in the spleen during non-diving periods when they could breathe and did not need elevated Hct for oxygen loading (Zapol et al., 1989; Castellini and Castellini, 1993). Elsner and Meiselman (1995) suggested that removing the cells from circulation except during diving might be an adaptive mechanism for the apparently high viscosity of seal blood.

Currently, we theorize that seal species that have relatively high viscosity or enhanced RBC aggregation will remove those cells from circulation via splenic sequestering during resting periods on the surface. However, the need for maximizing oxygen carrying capacity and delivery during diving must be greater than the cost of the increased viscosity, because some seal species appear to maximize Hct during diving bouts. Conversely, we hypothesize that seal species with very low blood viscosity and aggregation values (e.g., ringed seals), will keep Hct levels stable and will not remove the cells from circulation when at the surface and therefore not increase their Hct when diving.

\section{RBC HEMOGLOBIN CONCENTRATION}

Mean RBC hemoglobin concentration in humans are around 30 to $35 \mathrm{~g} / \mathrm{dl}$ but we have recorded MCHC values as high as 47 to $48 \mathrm{~g} / \mathrm{dl}$ in Weddell seals, an adaptation that increases blood oxygen carrying capacity per RBC (Kooyman, 1989; Elsner and Meiselman, 1995; Castellini et al., 2006). However, the rheological "cost" of the increased $\mathrm{MCHC}$ is an increase of cytoplasmic viscosity and the impairment of both membrane and cellular deformability: $\mathrm{RBC}$ containing high levels of $\mathrm{O}_{2}$ - binding proteins to guarantee adequate $\mathrm{O}_{2}$ supply thus seems to be of greater importance than possible rheological disadvantages. Nash and Meiselman $(1983,1991)$ have shown that when human RBC are dehydrated to a MCHC of about $50 \mathrm{~g} / \mathrm{dl}$, the cells change markedly in shape, show unrecoverable deformation and the cytoplasm behaves more like a solid. Interestingly, human blood cells with sickle cell disease are characterized by dehydrated RBC that exhibit increased MCHC (up to 50\%; Joiner, 1993). Let us assume that such similarly high $\mathrm{MCHC}$ values can only be reached in human cells under extreme disease stress and that this is the limit of $\mathrm{Hb}$ solubility in the RBC cytoplasm. Does this imply that some marine mammals have maximized about the same amount of $\mathrm{Hb}$ that can be held in solution in a mammalian RBC? This would make some adaptive sense. If they must maximize oxygen carrying capacity for diving then there are only two mechanisms to do so: (1) increase the Hct to maximum values during diving; (2) increase the amount of $\mathrm{Hb} /$ cell to its maximum. Both of these solutions have rheological costs associated with them. But the balance may have tipped more toward the advantage of carrying massive amounts of oxygen in the blood versus the rheological costs of moving such cells through the circulation.

While we know that Hct varies in diving mammals, no publication to date has evaluated whether MCHC levels change during diving. As part of a separate research project, we have recently examined blood patterns in Weddell seals at extremely elevated Hct levels of over $70 \%$ after very long and strenuous dives and have noted unusual RBC changes. If one assumes that the amount of hemoglobin per cell $(\mathrm{MCH})$ is constant over short time periods, then the relationship of whole blood Hb to Hct will be linear. That is, as Hct increases, then the total amount of $\mathrm{Hb}$ in the blood should increase in a linear fashion. However, as shown in Figure 3 for Weddell seals, this linear relationship no longer appears to hold at a Hct $>70 \%$ but rather begins to flatten. One possible interpretation of these data is an alteration in the RBC being released into the circulation at the limits of splenic constriction. Perhaps the RBC are much larger with the same total amount of $\mathrm{Hb} / \mathrm{cell}$, thus driving up Hct but not the amount of $\mathrm{Hb}$, or cells of the same size with less $\mathrm{Hb} /$ cell are being released. In either case, the rheological implications of extremely high Hct levels combined with potentially different populations of RBC are completely unknown.

It is clear that the dynamic relationships of Hct variability along with the theoretically static relationship of $\mathrm{Hb} / \mathrm{cell}$ are key points in understanding the rheological implications of diving in marine mammals. Some of this work can be conducted in vitro with collected blood samples, but other aspects of further studies in this area will need to be conducted in vivo with diving animals.

\section{NEONATAL DEVELOPMENT OF BLOOD OXYGEN DELIVERY}

Marine mammals show major developmental changes in diving physiology. Pups must develop their cardiovascular responses to diving along with blood and tissue oxygen stores, and subsequently are not able to dive as well as adults (Kooyman et al., 1983; Burns and Castellini, 1996; Burns et al., 2007). Burns et al. (2007) have shown that in some true seal species, Hct values of neonates do not change during development, while in other species, the Hct is elevated at birth, declines in the neonatal period and then increases as the animals mature and become better divers. Trillmich et al. (2008) have shown similar patterns in fur 


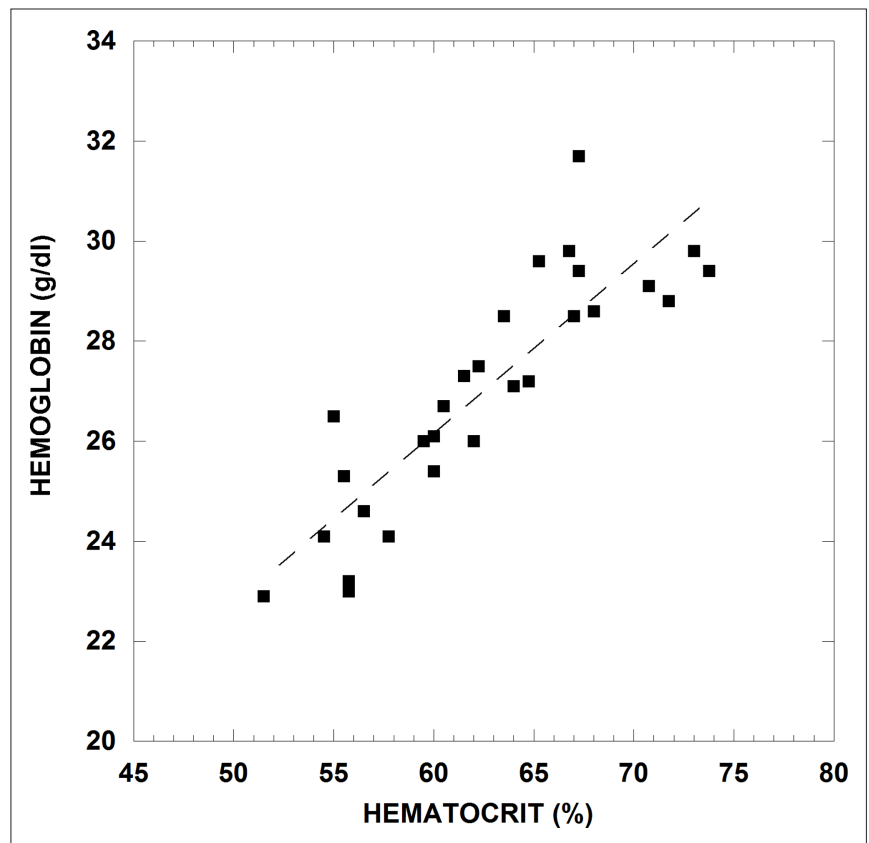

FIGURE 3 | Whole blood hemoglobin concentration versus hematocrit for blood from an adult Weddell seal during rest periods and after dives.

Venous blood samples were taken from an indwelling catheter after the seal surfaced from a natural dive into heparinized Vacutainers ${ }^{\odot}$ or syringes. An aliquot of whole blood was removed for spectrophotometric $\mathrm{Hb}$ analysis and the remaining blood immediately measured for Hct. This animal handling and sampling method has been well studied and documented for collecting diving recovery blood samples in Weddell seals (Kooyman et al., 1980; Qvist et al., 1986; Castellini et al., 1988).

seals and Weddell seals while Castellini et al. (1990) demonstrated that elephant seals are born with a Hct $(\sim 48-52 \%)$ that remains stable during the nursing period and then begins to increase at weaning to as high as $68-70 \%$. Because Hct is such a significant determinant of whole blood viscosity, varying patterns of Hct during development will impact native blood viscosity. To separate potential differences in inherent viscosity during development in seals, we have examined several rheological characteristics in young Weddell seals (Castellini et al., 2006). We found that blood from 2 - and 7-day-old pups demonstrated less aggregation than adult blood, but that at 7 days of age, the pups showed more aggregation than at 2 days. Also, adult Weddell seal blood showed significantly greater viscosity than pup blood at both native and standardized Hct values.

When considering the concept of oxygen delivery, it is informative to calculate the "RBC delivery effectiveness," defined as the ratio of Hct to blood viscosity and abbreviated as HVR. Castellini et al. (2006) have examined this parameter for Weddell seal pups and adults, and report that the HVR value is about 2-fold greater for Weddell pups than for adults, suggesting a more effective delivery of oxygen to the tissues. Subsequent increases of Hct and hence blood viscosity with pup maturation will tend to reduce the HVR, eventually leading to the lower values of HVR observed for adult Weddell seals.
This counter-intuitive finding for HVR in adult and pups again indicates the interplay of blood viscosity, Hct and the need for oxygen carrying capacity development in seals. It seems that even though the efficiency of oxygen delivery may be reduced at elevated Hct levels in adults, the advantage of increasing the volume of oxygen that can be carried by the blood is more critical to the animal than the efficiency at which it can be delivered. Because pups have a higher mass-specific metabolic rate, oxygen delivery to the tissues may need to be more efficient than in adults, which have a lower mass-specific tissue metabolic rate.

\section{IMPACT OF HIGH PRESSURE IN DIVERS}

While there have been no direct studies of high pressure on RBC rheological properties in marine mammals, RBC metabolism must continue to function well under natural diving at great depths. However, in human RBC, even mildly elevated pressure ( 9 ATM) can increase aggregation (Taylor et al., 1998). It has also been shown that slightly higher pressure ( $<15$ ATM) can alter the membrane lipid order, and therefore the fluidity of human RBC membranes (Chen et al., 1994; Barshtein et al., 1997). There is some evidence that the metabolic pathways of marine mammal RBC are either pressure insensitive or actually slightly enhanced in simulated diving to 2000 PSI (1360 m or 136 ATM of pressure) (Castellini et al., 2001, 2002), but the rheological characteristics of RBC were not examined in those studies. Differences of membrane properties between marine mammal and terrestrial RBC have been implicated in pressure tolerance differences, glucose transport properties and in the ability to count the cells using flow cytometry (Castellini et al., 1992, 2002; Williams et al., 2001). RBC membranes from several marine mammal species were found to have lower cholesterol and phospholipid content (Fayolle et al., 2000).

In a related study, platelet activation during pressurizationdecompression was tested in elephant seal blood. In contrast to human cells, seal platelets were not activated by simulated diving to 2800 PSI (190 ATM) (Field et al., 2001; Field, 2005) and the authors suggested this might be due to the elevated cholesterol content of seal platelets. While platelets are not significant factors in most rheological measurements of whole blood due to their lower concentration compared to RBC (about 1:20)and much smaller size, the possible relationships between pressure responses and membrane structure is an interesting finding in both platelets and RBC.

Investigation of how elevated hydrostatic pressure could impact flow characteristics of marine mammal RBC is essentially untouched. Even the most basic of experiments, such as measuring $\mathrm{RBC}$ aggregation rates under pressure, need to be conducted. When combined with the other changes in blood that occur during diving conditions (e.g., decreased $\mathrm{pH}$, variable Hct and low oxygen), the study of how blood rheology might change under elevated hydrostatic pressure becomes both fascinating and more complex. One current example of where such as study is relevant concerns the impact of SONAR on diving marine mammals and whether the absorbed energy from SONAR alters RBC properties. Several ex vivo studies have suggested that direct ensonification of terrestrial mammal tissues can result in bubble formation (Crum et al., 2005) and changes in blood properties (Shin et al., 2003). However, the impact of compression, rapid decompression 
(e.g., rapid ascents to the surface), simultaneous ensonification in SONAR ranges and comparative approaches to tissue selection (marine versus terrestrial mammals, deep versus shallow divers) have not been examined.

\section{FUTURE STUDIES RELEVANT TO COMPARATIVE PHYSIOLOGY}

The essential concepts of oxygen delivery and how changes in blood flow behavior could be altered by these environmental or physiological conditions extend far beyond terrestrial versus marine mammals. Rheological studies have been conducted on non-mammalian systems and become even more intriguing given the nucleated RBC of other vertebrates are not biconcave. For example, two important differences are that the RBC have a very low deformability compared to non-nucleated, biconcave RBC and that they have very low, if any, RBC aggregation properties (Usami et al., 1969, 1970; Waugh and Evans, 1976; Gaehtgens et al., 1981; Windberger and Baskurt, 2007).

It is clear, however, that we are far from a unifying principle of rheological patterns in vertebrate circulatory systems. There is no doubt that the flow behavior of blood is critical to the development

\section{REFERENCES}

Andrews, F. A., Korenek, N. L., Sanders, W. L., and Hamlin, R. L. (1991). Viscosity and rheologic properties of blood from clinically normal horses. Am. J. Vet. Res. 53, 966-971.

Barshtein, G., Bergelson, L., Dagan, A., Gratton, E., and Yedgar, S. (1997). Membrane lipid order of human red blood cells is altered by physiological levels of hydrostatic pressure. Am. J. Physiol. 272, H538-H543.

Baskurt, O. K., and Meiselman, H. J. (2003). Blood rheology and hemodynamics. Semin. Thromb. Hemost. 29, 435-450.

Baskurt, O. K., and Meiselman, H. J. (2007). Hemodynamic effects of red blood cell aggregation. Indian J. Exp. Biol. 45, 25-31.

Baskurt, O. K., and Meiselman, H. J. (2010). Lessons from comparative hemorheology studies. Clin. Hemorheol.Microcirc. 45, 101-108.

Baskurt, O. K., Hardeman, M. R., Rampling, M. W., and Meiselman, H. J. (eds) (2007). Handbook of Hemorheology and Hemodynamics. Amsterdam: IOS Press.

Baskurt, O. K., Marshall-Gradisnik, S., Payne, M., Simmonds, M., Brenu, E., Christy, R., and Meiselman, H. J. (2010). Assessment of hemorheological profile of koala and echidna. Zoology 113, 110-117.

Baskurt, O. K., Yalcin, O., and Meiselman, H. J. (2004). Hemorheology and vascular control mechanisms. Clin. Hemorheol. Microcirc. 30, 169-178.

Bauersachs, R. M., Wenby, R. B., and Meiselman,H.J.(1989).Determination of specific red blood cell aggregation indices via an automated system. Clin. Hemorheol. 9, 1-25.

Bull, B. S., and Brailsford, J. D. (1974). The zeta sedimentation rate. Blood 40, 550-557.

Burns, J. M., and Castellini, M. A. (1996). Physiological and behavioral determinants of the aerobic dive limit in Weddell seal pups (Leptonychotes weddellii). J. Comp. Physiol. B, Biochem. Syst. Environ. Physiol. 166, 473-483.

Burns, J. M., Lestyk, K. C., Folkow, L. P., Hammill, M. O., and Blix, A. S. (2007). Size and distribution of oxygen stores in harp and hooded seals from birth to maturity. J. Comp. Physiol. B, Biochem. Syst. Environ. Physiol. 177, 687-700.

Butler, P. J. (1982). Respiratory and cardiovascular control during diving in birds and mammals. J. Exp. Biol. 100, 195-221.

Castellini, J. M., and Castellini, M. A. (1993). Estimation of splenic volume and its relationship to long duration apnea in seals. Physiol. Zool. 66, 619-627.

Castellini, J. M., Castellini, M. A., and Kretzmann, M. B. (1990). Circulatory water balance in suckling and fasting northern elephant seal pups. J. Comp. Physiol. B, Biochem. Syst. Environ. Physiol. 160, 537-542.

Castellini, M. A., Castellini, J. M., and Kirby, V. L. (1992). Blood glucose handling methods can compromise analytical results: Evidence from marine mammals. J. Am. Vet. Assoc. 201, 145-148.

Castellini, M. A., Castellini, J. M., and Rivera, P. M. (2001). Adaptations to pressure in the RBC metabolism of

of systems that can change under conditions of elevated pressure, low blood flow, heterothermy, water balance and a wide range of other environmental and physiological stresses that are seen across the vertebrate groups. Comparative studies across species to test the interactions of Hct, RBC hemoglobin content, cell shape, cell protein level, viscosity and aggregation would be extremely valuable. Hopefully, through this review, we have brought to the forefront some of the basic questions that can be asked in marine versus terrestrial comparisons and have provided the framework for broader explorations in the area of comparative rheology.

\section{ACKNOWLEDGMENTS}

Research on Weddell seals was supported by National Science Foundation OPP grant 0130417 to Michael A. Castellini and supported in the Antarctic by Raytheon Polar Services Company. Seals were captured and handled under MMPA permit \#1033-1683 issued to Michael A. Castellini and approved under UAF Institutional Animal Care and Use Committee Protocols. The study was supported in part by the Akdeniz University Research Fund and by NIH Awards HL15722, HL70595 and TW001295.

diving mammals. Comp. Biochem. Physiol. 129, 751-757.

Castellini, M.A., Costa, D. P., and Huntley, A.C. (1986). Hematocrit variation during sleep apnea in elephant seal pups. Am. J. Physiol. 251, R429-R431.

Castellini, M. A., Davis, R. W., and Kooyman, G. L. (1988). Blood chemistry regulation during repetitive diving in Weddell seals. Physiol. Zool. 61, 379-386.

Castellini, M. A., Elsner, R., Baskurt, O. K., Wenby, R. B., and Meiselman, H. J. (2006). Blood rheology of Weddell seals and bowhead whales. Biorheology 43, 57-69.

Castellini, M. A., Rivera, P. M., and Castellini, J. M. (2002). Biochemical aspects of pressure tolerance in marine mammals. Comp. Biochem. Physiol. 133A, 893-899.

Chen, S., Gavish, B., Barshtein, G., Mahler, Y., and Yedgar, S. (1994). Red blood cell aggregability is enhanced by physiological levels of hydrostatic pressure. Biochim. Biophys. Acta 1192, 247-252.

Chien, S. (1975). "Biophysical behavior of red cells in suspension," in The Red Blood Cell, ed. D. M. Surgenor (New York: Academic Press), 1031-1133.

Chien, S. (1987). Red cell deformability and its relevance to blood flow. Annu. Rev. Physiol. 49, 177-192.

Chien, S., Usami, S., Dellenback, R. J., and Bryant, C. A. (1971). Comparative hemorheology-hematological implications of species differences in blood viscosity. Biorheology 8, 35-57.

Cokelet, G. R., and Meiselman, H. J. (2007). "Macro and micro rheological properties of blood," in Handbook of Hemorheology and Hemodynamics, eds. O. K. Baskurt, M. R. Hardeman, M. W. Rampling, and H. J. Meiselman (Amsterdam: IOS Press), 45-71.

Crum, L.A., Bailey, M. R., Guan, J., Hilmo, P. R., Kargl, S. G., Matula, T. J., and Sapazhnikov, O.A. (2005). Monitoring bubble growth in supersaturated blood and tissue ex vivo and the relevance to marine mammal bioeffects. Acoust. Res. Lett. Online 6, 214-220.

Elsner, R., and Meiselman, H. J. (1995). Splenic oxygen storage and blood viscosity in seals. Mar. Mamm. Sci. 11, 93-96.

Elsner, R., Meiselman, H. J., and Baskurt, O. K. (2004). Temperature-viscosity relations of bowhead whale blood: a possible mechanism for maintaining cold blood flow. Mar. Mamm. Sci. 20, 339-344.

Elsner, R. W., and Gooden, B. (1983). Diving and Asphyxia: A Comparative Study of Animalsand Man.Physiological Society Monograph 40. Cambridge: Cambridge University Press.

Engen, R. L., and Clark, C. L. (1990). High performance liquid chromatography determination of erythrocyte membrane phospholipid composition in several animal species. Am. J. Vet. Res. 51, 577-580.

Eylar, E. H., Madoff, M. A., Brody, O. V., and Oncley, J.L. (1962). The contribution of sialic acid to the surface charge of the erythrocyte. J. Biol. Chem. 237, 1992-2000.

Fayolle, C., Leray, C., Ohlmann, P., Gutbier, G., Cazenave, J. P., Gachet, C., and Groscolas, R. (2000). Lipid composition of blood platelets and erythrocytes of southern elephant 
seal (Mirounga leonina) and antarctic fur seal (Arctocephalus gazella). Comp. Biochem. Physiol. B 126, 39-47.

Field, C. (2005). A Biophysical Characterization of Northern Elephant Seal (Mirounga angustirostris) Blood Platelets and Response to Temperature and Pressure Changes. $\mathrm{PhD}$ Thesis, University of California, Davis.

Field, C. L., Walker, N. J., and Tablin, F. (2001). Northern elephant seal platelets: analysis of shape change and response to platelet agonists. Thromb. Res. 101, 267-277.

Gaehtgens, P., Will, G., and Schmidt, F. (1981). Comparative rheology of nucleated and non-nucleated red blood cells. Pflugers Arch. 390, 278-282.

Gascoyne, S. C., and Hawkey, C. M. (1992). Patterns of variation in vertebrate haematology. Clin. Hemorheol. 12, 627-637.

Guard, C. L., and Murrish, D. E. (1975). Effects of temperature on the viscous behavior of blood from Antarctic birds and mammals. Comp. Biochem. Physiol. 52A, 287-290.

Hawkey, C. M. (1975). Comparative Mammalian Haematology. London: William Heinemann Medical Books Ltd.

Hedrick, M. S., and Duffield, D. A. (1991). Hematological and rheological characteristics of blood in 7 marine mammal species - physiological implications for diving behavior. J. Zool. 225, 272-283.

Hedrick, M.S., Duffield,D.A., and Cornell, L. H. (1986). Blood-viscosity and optimal hematocrit in a deep-diving mammal, the northern elephant seal (Mirounga angustirostris). Can. J.Zool. 64, 2081-2085.

Joiner, C. H. (1993). Cation transport and volume regulation in sickle red blood cells. Am. J. Physiol. 264, C251-C270.

Johnn, H., Phipps, C., Gascoyne, S. C., Hawkey, C., and Rampling, M. W. (1992).A comparison of the viscometric properties of the blood from a wide range of mammals. Clin. Hemorheol. 12, 639-647.

Kooyman, G. L. (1989). Diverse divers: physiology and behavior.Zoophysiology 23, 1-200.

Kooyman, G. L., Castellini, M. A., Davis, R. W., and Maue, R. A. (1983). Aerobic diving limits of immature Weddell seals. J. Comp. Physiol. 151, 171-174.

Kooyman, G. L., Wahrenbrock, E. A., Castellini, M. A., Davis, R. W., and
Sinnett, E. E. (1980). Aerobic and anaerobic metabolism during voluntary diving in Weddell seals: evidence of preferred pathways from blood chemistry and behavior. J. Comp. Physiol. 138, 335-346.

Kraft,W., and Dürr, U.M. (1997). Klinische Labordiagnostik in der Tiermedizin. Stuttgart, New York: Schattauer.

Meiselman, H. J. (1993). Red blood cell role in RBC aggregation: 1963-1993 and beyond. Clin. Hemorheol. 13, 575-592.

Meiselman, H. J., Castellini, M. A., and Elsner, R. (1992). Hemorheological behavior of seal blood. Clin. Hemorheol. 12, 657-675.

Meiselman, H. J., Neu, B., Rampling, M. W., and Baskurt, O. K. (2007). RBC aggregation: laboratory data and models. Indian J. Exp. Biol. 45, 9-17.

Nash,G.B., and Meiselman,H.J.(1983).Red cell and ghost viscoelasticity - effects of hemoglobin concentration and in vivo ageing. Biophys. J. 43, 63-73.

Nash, G. B., and Meiselman, H. J. (1991). Effect of dehydration on the viscoelastic behavior of red cells. Blood Cells 17, 517-522.

Neu, B. J., and Meiselman, H. J. (2007). "Red blood cell aggregation," in Handbook of Hemorheology and Hemodynamics, eds. O. K. Baskurt, M. R. Hardeman, M. W. Rampling, and H. J. Meiselman (Amsterdam: IOS Press), 114-136.

Oulevey, J., Bodden, E., and Thiele, O. W. (1977). Quantitative determination of glycosphingolipids illustrated by using erythrocyte membranes of various mammalian species. Eur. J. Biochem. 79, 265-267.

Qvist, J., Hill, R. D., Schneider, R. C., Falke, K. J., Liggins, G. C., Guppy, M., Elliot, R. L., Hochachka, P. W., and Zapol, W. M. (1986). Hemoglobin concentrations and blood gas tensions of freediving Weddell seals. J. Appl. Physiol. 64, 1560-1569.

Rampling, M. W., Meiselman, H. J., Neu, B., and Baskurt, O. K. (2004). Influence of cell-specific factors on red blood cell aggregation. Biorheology 41, 91-112.

Scholander, P. F. (1940). Experimental investigations on the respiratory function in diving mammals and birds. Hvalradets Skrifter 22, 1-131.

Shin, S., Ku, Y., Suh, J.-S., Moon, S.-Y., and Jang, J.-Y. (2003). Characteristics of blood flow resistance under transverse vibration: red blood cell suspension in dextran-40. Ann. Biomed. Eng. 31, 1077-1083.
Sowemimo-Coker, S. O., Whittingstall, P., Pietsch, L., Bauersachs, R. M., Wenby, R. B., and Meiselman, H. J. (1989). Effects of cellular factors on the aggregation behavior of human, rat and bovine erythrocytes. Clin. Hemorheol. 9, 723-737.

Stoltz, J. F., Singh, M., and Riha, P. (1999). Hemorheology in Practice. Amsterdam: IOS Press.

Taylor, W. F., Chen, S., Barshtein, G., Hyde, D. E., and Yedgar, S. (1998). Enhanced aggregability of human red blood cells by diving. Undersea Hyperb. Med. 25, 167-70.

Trillmich, F., Rea, L., Castellini, M. A., and Wolf, J. B. W. (2008). Agerelated changes in hematocrit in the Galápagos sea lion (Zalophus wollebaeki) and the Weddell seal (Leptonychotes weddellii). Mar. Mamm. Sci. 24, 303-314.

Usami, S., Chien, S., and Gregersen, M. I. (1969). Viscometric characteristics of blood of the elephant, man, dog, sheep and goat. Am. J. Physiol. 217, 884-890.

Usami, S., Magazinovic, V., Chien, S., and Gregersen, M. I. (1970). Viscosity of turkey blood: rheology of nucleated erythrocytes. Microvasc. Res. 2 489-499.

Uyuklu, M., Meiselman,H. J., and Baskurt, O. K. (2007). Effect of decreased plasma cholesterol by atorvastatin treatment on erythrocyte mechanical properties. Clin. Hemorheol. Microcirc. 36, 25-33.

Wang, D. (1994). Band 3 Protein: structure, flexibility and function. FEBS Lett. 346, 26-31.

Waugh, R., and Evans, E. A. (1976). Viscoelastic properties of erythrocyte membranes of different vertebrate animals. Microvasc. Res. 12, 291-304.

Wickham, L. L., Bauersachs, R. M. Wenby, R. B., Sowemimo-Coker, S., Meiselman, H. J., and Elsner, R. (1990). Red cell aggregation and viscoelasticity of blood from seals, swine and man. Biorheology 27, 191-204.

Wickham, L. L., Elsner, R., White, F. C., and Cornell, L. H. (1989). Blood viscosity in phocid seals: possible adaptations to diving. J. Comp. Physiol. B, Biochem. Syst. Environ. Physiol. 159, 153-158.

Williams, E. E., Stewart, B. S., Beuchat, C. A., Somero, G. N., and Hazel, J. R. (2001). Hydrostatic-pressure and temperature effects on the molecular order of erythrocyte membranes from deep-, shallow-, and non-diving mammals. Can. J. Zool. 79, 888-894.

Windberger, U., and Baskurt, O. K. (2007). "Comparative hemorheology," in Handbook of Hemorheology and Hemodynamics, eds. O. K. Baskurt, M. R. Hardeman, M. W. Rampling, and H. J. Meiselman (Amsterdam, Berlin, Oxford, Tokyo, Washington DC: IOS Press). 267-285.

Windberger, U., Bartholovitsch, A., Plasenzotti, R., Korak, K. J., and Heinke, G. (2003). Whole blood viscosity, plasma viscosity and erythrocyte aggregation in nine mammalian species: reference values and comparison of data. Exp. Physiol. 88, 431-440.

Wong, P. (1999). A basis of echinocytosis and stomatocytosis in the disc-sphere transformations of the erythrocyte. $J$. Theor. Biol. 196, 343-361.

Zapol, W. M., Hill, R. D., Qvist, J., Falke, K., Schneider, R. C., Liggins, G. C., and Hochachka, P. W. (1989). Arterial gas tensions and hemoglobin concentrations of the freely diving Weddell seal. Undersea Biomed. Res. 16, 363-73.

Zapol, W. M., Liggins, G. C., Schneider, R. C., Qvist, J., Snider, M. T., Creasy, R. K., and Hochachka, P. W. (1979). Regional blood flow during simulated diving in the conscious Weddell seal. J. App. Physiol. 47, 968-973.

Conflict of Interest Statement: The authors declare that the research was conducted in the absence of any commercial or financial relationships that could be construed as a potential conflict of interest.

Received: 11 May 2010; accepted: 18 October 2010; published online: 02 December 2010.

Citation: Castellini MA, Baskurt O, Castellini JM and Meiselman HJ (2010) Blood rheology in marine mammals. Front. Physio. 1:146. doi: 10.3389/ fphys.2010.00146

This article was submitted to Frontiers in Aquatic Physiology, a specialty of Frontiers in Physiology.

Copyright (c) 2010 Castellini, Baskurt, Castellini and Meiselman. This is an open-access article subject to an exclusive license agreement between the authors and the Frontiers Research Foundation, which permits unrestricted use, distribution, and reproduction in any medium, provided the original authors and source are credited. 